\title{
DYNAMICS OF PATTERNS AND INTERFACES IN SOME REACTION-DIFFUSION SYSTEMS FROM CHEMICAL AND BIOLOGICAL VIEWPOINTS
}

\author{
MASAYASU MIMURA*
}

1. Initiation to reaction-diffusion systems. In order to understand spatiotemporal patterns arising in diffusing medium, reaction-diffusion (RD) equation models have been discussed not only in applied sciences but also in mathematics: In most applications, the models are simply described by

$$
U_{t}=D \Delta U+F(U) \quad t>0, x \in \Omega,
$$

where $U=\left(u_{1}, u_{2}, \cdots, u_{N}\right), D$ is a diagonal diffusion matrix with elements $\left\{d_{i}\right\}$ where $d_{i}$ is non-negative consasnt $(i=1,2, \cdots, N)$. $\Omega$ is a bounded domain in $R^{n} . \Delta$ is the Laplacian operator in $R^{n} . F(U)=\left(F_{1}(U), F_{2}(U), \cdots, F_{N}(U)\right)$. In the absence of the reaction terms $F(U),(1-1)$ is decoupled so that each of the variables reduces to the following heat equation for a scalar unknown $u$

$$
u_{t}=d \Delta u \quad t>0, x \in \Omega .
$$

For a fundamental result on (1-1) with the Neumann boundary conditions, we have the following result: Suppose that there is a compact positively invariant set $S$ such that $U(0, x)$ is in $S$ for any $x \in \Omega$. Then any solution $U(t, x)$ tends to be asymptotically homogeneous if the diffusion matrix $D$ is suitably large[1].

This result is intuitively obvious, because the dynamics of solutions is dominantly governed by large diffusion. It indicates that the asymptotic behavior of solutions of (1-1) can be known by the analysis of the corresponding ODEs

$$
U_{t}=F(U) \quad t>0 \text {. }
$$

Therefore, the essential problem of (1-1) should be the case when $D$ is not necessarily large. Along this line, let us first consider the scalar case $(N=1)$ of $(1-1)$, that is

$$
u_{t}=d \Delta u+f(u) \quad t>0, x \in \Omega .
$$

Some qualitative properties of solutions of (1-4) with the Neumann boundary condition are already known as follows:

(i) Stable attractors consist only of equilibrium solutions only[2];

(ii) When the domain $\Omega$ is convex, any non-constant equilibrium solutions are unstable, even if they possibly exist[3],[4];

(iii) There are some non-convex domain $\Omega$ and $f(u)$ such that there are stable non-constant equilibrium solutions[4].

${ }^{*}$ Department of Mathematical and Life Sciences, Institute for Nonlinear Sciences and Applied Mathematics, Graduate School of Science, Hiroshima University, Japan (mimura@math.sci.hiroshima-u.ac.jp). 
The results of (i) and (ii) indicate that if $\Omega$ is restricted to be convex, any bounded solution of (1-4) generically approaches one of the stable spatially constant equilibrium solutions which are given by stable critical points of the diffusinless equation of (1-4)

$$
u_{t}=f(u) \quad t>0 \text {. }
$$

This result also seems to be obvious, because the equation (1-4) is still a diffusion equation. For this reason, it had long believed that RD systems of the form (1-1) are less interesting from the viewpoints of qualitative properties of solutions, comparing with other types of PDEs inclding the Navier-Stokes equation, MHD equations and so on.

1.1. Paradox of diffusion. In 1952, two surprising paradoxical evidences of "diffusion" were demonstrated by using RD models. The first contributor is a mathematician, A. Turing[5] who stated that diffusion enhances spatial inhomogeneities. In order to show this paradox, he proposed a one-dimensional reaction-diffusion system for two unknowns $(a, i)(t, x)$ in the interval $I$

$$
\begin{aligned}
& a_{t}=d_{a} a_{x x}+f(a, i) \\
& i_{t}=d_{i} i_{x x}+g(a, i),
\end{aligned} \quad t>0, x \in I
$$

where $a$ and $i$ are the concentrations of chemical species, which play arole of an activator and its inhibitor, respectively. Both species are diffusive. For the ineteraction of $a$ and $i, f$ and $g$ are specified by the follwing ansatz:

(a1) Activator increases in an autocatalytic way;

(a2) Activator produces Inhbitor at the same time;

(a3) Inhibitor inhibits the increasing of Activator.

One specific form of thefunctions $f$ and $g$ to include the interaction (a1) (a3) is

$$
\begin{aligned}
& f(a, i)=a(1-a)\left(a-a_{0}\right)-I \\
& g(a, i)=a-p i+q,
\end{aligned}
$$

where $a_{0}\left(0<a_{0}<1\right), p(p>0)$ and $q$ are all constants. Turing's claim is that If Inhibitor diffuses much faster than Activator does $\left(d_{a}<d_{i}\right)$, then spatial inhomogeneites of $a$ and $i$ is possibly enhanced by diffusion. The mathematical tool is the instability argument on the spatially constant equilibrium solution of (1-6).

The second contributors are two neurophysilogists, A. L. Hodgkin and A. F. Huxley. They observed in experiments that nerve impulse propagates with constant shape and constant speed along the axon, as if it were a pulse wave. In 1952, they proposed a PDE model of the squid axon which describes excitation and propagation of the impulse. It is given by the following single RD equation supplemented with three ODEs:

$$
\begin{aligned}
V_{t} & =d V_{x x}+f(V, m, n, h), \\
m_{t} & =M(m, V) \\
h_{t} & =H(h, V) \\
n_{t} & =N(n, V),
\end{aligned}
$$


where $V$ is the membrane potential and $m, h$ and $n$ are conductance variables. The nonlinearities $f, M, H$ and $N$ are suitable functional forms (see[6]). In those days, it had not been believed that diffusion equations could generate such propagating waves. However, it was numerically demonstrated that the system (1-8) possessed a travelling pulse solution, which could predict a wave form of the normal propagating impulse and its velocity was in good agreement with experimental measurements.

Around 10 years later, FitzHugh[7] and independently Nagumo[8] derived a simplified version of the $\mathrm{H}-\mathrm{H}$ equation without loosing qualitative by essential properties of solutions. It is described by

$$
\begin{aligned}
& u_{t}=d u_{x x}+u(1-u)(u-a)-v \\
& v_{t}=\varepsilon^{-1}(u-p v+q),
\end{aligned}
$$

where $u$ corresponds to the membrane potential $V$ and $\varepsilon$ is a small positive constant. (1-9) is called the FitzHugh-Nagumo (FHN) equation. Here we emphasize that (1-6) with (1-7) and (1-9) are exactly the same nonlinear forms in a sense that $u$ and $v$ play a role of activator and its inhivitor, respectively.

We thus find that two paradoxes of diffusion such as

(i) Diffusion enhances spatial inhomogeneity;

(ii) Diffusion causes a localized pulse wave which moves with constant velocity

can be explained by the two component activator-inhibitor RD systems.

Therefore, I would like to say that RD systems were born in 1952 from pattern formation viewpoints. Since then, RD systems of the form (1-1) have been discussed in the fields of not only applied sciences but also mathematics. Especially, One main problem on (1-1) is to understand what kind of unexpected patterns can be generated by the interplay of diffusion and reaction. In this lecture, we restrict ourselves to a special class of RD systems in order to consider this problem.

2. Resource-consumer RD systems. 'Among several classes of RD systems from pattern formation viewpoints, we will discuss a class of systems which describe the interaction between "consumer" and "its resource" such as bacteria-nutirient and combustion-chemical reactant. They are generally of the form

$$
\begin{array}{ll}
u_{t} & =d_{u} \Delta u+h(u) f(v) \\
v_{t} & =d_{v} \Delta v-h(u) f(v),
\end{array} \quad t 0, x \in \Omega
$$

where $u$ and $v$ denote respectively the densities of a consumer and its resource. $h(u) f(v)$ is the growth term of the consumer, where $f(v)$ and $h(u)$ are respectively positive functions with $v$ and $u$. One easily notices that $u$ is always gorws, while $v$ is always consumed. One typical feature of this system is that the same nonlinearity $h(u) f(v)$ is included so that there is the balance law of the total density of $u$ and $v$. (2-1) may be slightly modified as

$$
\begin{array}{ll}
u_{t} & =d_{u} \Delta u+h(u) f(v)-\gamma u \\
v_{t} & =d_{v} \Delta v-h(u) f(v),
\end{array} \quad t 0, x \in \Omega
$$

where $\gamma$ is the death ( or removal) rate which is a positive constant. Let us introduce some models which are described by $(2-1)$ or $(2-2)$. 
2.1. A diffusion model of bacterial colonies. The diversity and complexity of spatio-temporal patterns arising in biological systems often take place through the intertwinement of complex biological factors and environmental conditions. Intensive investigations have shown that the growth of bacterial colonies produces various complex patterns, depending on the species and environmental conditions. For instance, the bacterial species called Bacillus subtilis is known to exhibit qualitatively different two-dimensional colony patterns as a result of growth and cell division on the surface of thin agar plates by feeding on nutrients[9](Fig. 1). Colony patterns change drastically when the concentrations of agar and nutrient, say $C_{a}$ and $C_{n}$, are globally varied. They are classified qualitatively into five types, each of which is observed in the regions labeled $\mathrm{A}-\mathrm{E}$ in the $\left(C_{a}, C_{n}^{-1}\right)$-plane. In the region $\mathrm{A}$ (hard agar medium with poor nutrient), colony patterns exhibit tip-splitting growth with characteristically branched structures(Fig. 2). These patterns are similar to those observed in diffusion limited processes in solidification from a supersaturated solution, solidification from an undercooled liquid, and electro-chemical deposition. Also, the structures are clearly reminiscent of two-dimensional diffusion-limited aggregation (DLA) clusters, which can be generated by a simple particle model for randomly branched patterns grown through diffusion-limited processes. Increasing $C_{n}$ with high fixed $C_{a}$ values (corresponding to the change from the region $\mathrm{A}$ to $\mathrm{B}$ ), the branch thickness of the colony increases gradually and colony patterns eventually become Eden-like. For high $C_{n}$ and low $C_{a}$ (soft agar medium with rich nutrient) in the region $\mathrm{D}$, colony patterns drastically change to be spread homogeneously, which look macroscopically like a perfect disk. It is likely that the movement of bacterial cells inside a colony can be described in terms of diffusion, and there is no microscopic branching at all. In the region $\mathrm{E}$, between the regions $\mathrm{A}$ and $\mathrm{D}$, there emerge colony patterns clearly reminiscent of the so-called dense-branching morphology (DBM). Though the branching is very dense, the advancing envelope looks characteristically smooth compared with DLA-like colonies in the region A. Finally, in the region $\mathrm{C}$, between the regions $\mathrm{B}$ and $\mathrm{D}$, colonies spread and rest alternately, leaving stationary concentric ring-like patterns. The above experimental observations lead to the following naive question:

Is the diversity of colony patterns observed in experiments caused by different effects or governed by the same underlying mechanisms?

For modelling of bacterial colonies, RD equation models for nutrient-limited growth of bacteria have been proposed. Let $b(t, x), n(t, x)$ be the population density of bacterial cells and the concentration of nutrients at time $t$ and position $x$. The first model is described by

$$
\begin{aligned}
& b_{t}=d \Delta b+\omega g(n) b, \\
& n_{t}=\Delta n-g(n) b,
\end{aligned} \quad t 0, x \in \Omega
$$

where $g(n)$ is the growth rate[20]. A simple form is the Malthusian rate $g(n)=n$. $\omega$ is the conversion rate of growth. Without loss of generality, we may put $\omega=1$. The initial conditions to (2-3) are

$$
\begin{aligned}
& b(0, x)=b_{0}(x), \\
& n(0, x)=n_{0},
\end{aligned} \quad t 0, x \in \partial \Omega
$$

where, from the biological requirement, $b_{0}(x)$ is a delta function-like distribution of the initial density of the bacteria and $n_{0}$ is the initial concentration of nutrients which 
is distributed uniformly in space. We remark that $C_{a}$ and $C_{n}$ correspond to $d^{-1}$ and $n_{0}$, respectively. When $d^{-1}$ and $n_{0}$ are globally varied, what kind of patterns do the problem (2-3), (2-4) generate? It is numerically shown that the resulting pattern of (2-3), (2-4) exhibit two cases, depending on the values of $n_{0}$ only: One is the extinction of bacteria for small $n_{0}$ and the other is that colony expands uniformly, taking a disk shape, for large $n_{0}$. This pattern is similar to the one in the region $\mathrm{D}$.

In order to modify the model (2-3), we propose a phenomenological time-space continuum model for the density of bacterial cells and the concentration of nutrients at the macroscopic level[10]. The essential ansatz in our modelling is the introduction of the internal state in each cell, so that if this state variable is increasing, then the bacteria actively move, grow and perform cell-division, while if it is decreasing, then it does nothing at all. In order to model this situation in a simple way, we assume that the bacterial cells consist of two types; active cells and inactive ones. In fact, a group of actively moving cells clearly are observed in the tip of each growing finger in the region $\mathrm{E}$, as if they were a finger nail. They seem to drive the growth of the finger tip, leaving inactive cells behind. Let $b(t, x)$ and $w(t, x)$ be the densities of the active and inactive cells, respectively, (hence the sum $b(t, x)+w(t, x)$ is the density of total bacterial cells) and $n(t, x)$ be the concentration of nutrients. The model for $b, n$ and $w$ is given by

$$
\begin{array}{rlr}
b_{t} & =\nabla(d \nabla b)+n b-a(b, n) b, & \\
n_{t} & =\Delta n-n b & \\
w_{t} & =a(b, n) b, &
\end{array}
$$

where $d=d(k)$ is the ratio of the diffusion rates of the bacterial cells and the nutrient. Here $k$ is the parameter corresponding to the inverse of the agar concentration. We make a plausible assumption that $d(k)$ is monotone increasing with $k$. Here we take a simple form of $d(k)$ is $d(k)=d_{0} k$ for some positiveconstant $d_{0}$. The rate of change from the active cells to the inactive ones is $a(b, n)$, which has to be specified and is caused by the mechanism of the internal state. We neglect the rate of change from the inactive cells to the active ones, because it is observed that once active cells become inactive, they never become active again unless food is added artificially. It is plausible that $a(., n)$ decreases with an increase in nutrient concentration $n$. However, the dependency of the active cell population $b$ on $a(b,$.$) is unclear. We observe that if$ the cell population $b$ becomes quite small, each cell is not very active. It is therefore assumed that $a(b,$.$) is a decreasing function of b$. Hereafter for concreteness and simplicity, we adopt the functional form $a(b, n)=a_{0}(1+b)^{-1}(1+n)^{-1}$ with constant $a_{0}$. It should be stressed, however, that this particular form is not essential to our conclusion below, and other functions which decrease with $b$ and $n$ well work as well. The first two equations of (2-5) are closed for $b$ and $n$, so, the unknown variable $w$ can be obtained in terms of them.

The initial and boundary conditions for $(2-5)$ are

$$
\begin{aligned}
& b(0, x)=b_{0}(x), \\
& n(0, x)=n_{0}, \\
& w(0, x)=0,
\end{aligned} \quad t>0, x \in \Omega
$$


where $b_{0}(x)$ is a delta function-like distribution of the initial concentration of the active cells, and $n_{0}$ is the initial concentration of nutrients which is distributed uniformly in space and

$$
\frac{\partial b}{\partial \nu}=\frac{\partial n}{\partial \nu}=0 \quad t>0, x \in \partial \Omega,
$$

where $\nu$ is the outerward normal unit vector on $\partial \Omega$. We note that the parameters $\left(n_{0}, k\right)$ in the model $(2-5),(2-6)$ correspond to $\left(C_{n}, C_{a}^{-1}\right)$ in the experiments. We first show the result on asymptotitc behavior of solutions of $(2-5) \sim(2-7)$.

Theorem 1. Consider the problem (2-5) (2-7). There is some constant $n_{\infty}>0$ and some function $w_{\infty}(x) \geq 0$ such that

$$
\lim _{t \rightarrow \infty}(n, b, w)(t, x)=\left(n_{\infty}, 0, w_{\infty}(x)\right)
$$

This thorem implies that $u$ and $v$ become spatially homogeneous asymptotically. From a biological viewpoint, we needs to know the spatio-temporal behavior of the total bacterial cells $b+w$. We thus find $\lim _{t \rightarrow \infty}(b+w)(t, x)=w_{\infty}(x)$. It indicates that what we should know is the spatial profile of $w_{\infty}(x)$. However, we have not yet provide any analytical method for this problem, so that we have to relay on numerical procedures. Let us numerically consider $(2-5) \sim(2-7)$ in two-dimensions. For quite small $n_{0}$ and $k$ (hard and poor environment), it is shown that $b(t, x)$ consists of many spots, each of which splits into two smaller ones as time goes on. This process implies the tip-splitting phenomenon. The resulting pattern of $v+w$ exhbits DLA-like strucutre(Fig. 3). Increasing $d$ with approximately the same value of $n_{0}$, the pattern changes to a DBM-like one. On the other hand, for large $k$ and $n_{0}$ (soft and rich environment), $b$ exhibits an expanding ring pattern where no unstable mechanism occurs so that the pattern of $b+w$ simply forms an expanding disk which is similar to the ones in region $\mathrm{D}$. If $k$ is decreasing with fixed large $n_{0}$, a pattern of growth and no growth occur alternately and the ever-expanding disk pattern changes to an immobilized concentric-ring pattern. In spite of the simplicity of the system, one finds that the model $(2-5) \sim(2-7)$ reproduces four of the different patterns corresponding to those in the regions $\mathrm{A}, \mathrm{C}, \mathrm{D}$, and $\mathrm{E}$, depending on values of $k$ and $n_{0}$. Thus, summarizing the above results, our reaction-diffusion model exhibits the phase diagram of colony patterns except fro the region $\mathrm{B}$.

For Eden-like patterns in the region B (hard agar medium with rich nutrient), experimental observations indicate that bacterial cells hardly move, but cells grow moderately because of an adequate supply of nutrient, so that the resulting colony pattern expands due to a mechanism different from the others. In order to model this situation, we have to replace $d(k)$ with a bacterial population-dependent diffusion term $d=d_{1} k(b+w)$ with positive constant $d_{1}$. The resulting modified system exhibits Eden-like pattern.

We thus find that a simple RD system model (2-9) can reproduce five different types of patterns which seem to be similar the ones observed in experiments when the parameters are varied globally. Here it should be emphasized that patterns are formed by the inactive cells $w$, which record the history of the active cells $u$.

2.2. Autocatalytic reaction-diffusion model. Motivated by the bacterial colony model, we consider the following autocatalytic chemical reaction processes: 


$$
\begin{aligned}
m V+U & \rightarrow(m+1) V \\
n V & \rightarrow W
\end{aligned}
$$

where $m$ and $n$ are positive intergers. Let $u, v$ and $w$ be the concentrations of the species $U, V$ and $W$. Although this system does not possess resource-consumer interaction, the model can be described by

$$
\begin{aligned}
& u_{t}=d_{u} \Delta u-u v^{m} \\
& v_{t}=d_{v} \Delta v+u v^{m}-a v^{n},
\end{aligned} \quad t>0, x \in \Omega
$$

where $d_{u}$ and $d_{v}$ are diffusion rates of the chemical species $u$ and $v$, respectively and $a$ is a positive constant. However, When $m=2$ and $n=1,(2-8)$ is called the GrayScott model without feed process[11]. If the species $W$ is immobile, it is analogous to the inactive cells in bacterial colony model so that the concentration of $W$, say $w$, is simply given by

$$
w_{t}=a v \quad t>0, x \in \partial \Omega
$$

The boundary conditions are

$$
\frac{\partial u}{\partial \nu}=\frac{\partial v}{\partial \nu}=0 \quad t>0, x \in \partial \Omega
$$

where $\nu$ is the outerward normal unit vector on the boundary $\partial \Omega$. In a similar way to $(2-6)$, the initial conditions are given as

$$
u(0, x)=u_{0}>0, v(0, x)=v_{0}(x) \geq 0, w(0, x) \equiv 0 \quad x \in \Omega .
$$

The first implies that the initial reactant of $u$ is uniformly distributed in the vessel $\Omega$. Because of no feeding process, we may say that (2-8) falls into the framework of closed systems, which are quite different from open systems (3-12) with feeding process. One could expect that any closed systems do not generally exhibit any spatial patterns asymptotically. This expectation is true for the problem $(2-8) \sim(2-11)$. The result is shown as follows:

Theorem 2[12]. Consider the problem (2-8), (2-10), (2-11).

(i) For $n \geq m, \quad \quad \lim _{t \rightarrow \infty}(u(t, x), v(t, x))=(0,0)$;

(ii) For $n<m$, there is some constant $u_{\infty}>0$, depending on both $u_{0}$ and $v_{0}(x)$, such that

$$
\lim _{t \rightarrow \infty}(u(t, x), v(t, x))=\left(u_{\infty}, 0\right) .
$$

By this theorem, we had cocluded that the problem (2-8), (2-10), (2-11) is less interesting from pattern formation viewpoints. However, we address teh following question: how is the behavior of the thrid variable $w$ which is given by 


$$
w(t, x)=a \int^{t} u(s, x) d s, \quad t>0, x \in \Omega,
$$

that is, the time-history of $u$. It is also proved that there is an equlibirium state such that

$$
\lim _{t \rightarrow \infty} w(t, x)=w_{\infty}(x) \geq 0 .
$$

However, we do not obtain any information on the spatial patterns of $w_{\infty}(x)$. Taking $d$ and $u_{0}$ as free parameters, we numerically consider the behavior of solutions $(u, v, w)$ to the problem $(2-8) \sim(2-11)$ with $m=2$ and $n=1$ in a rectangular domain. When $d$ and $u_{0}$ are both suitably large, $v(t, x)$ exhibits a ring pattern which expands uniformly and the corresponding $w(t, x)$ exhibits an expanding disk-like pattern. As $d$ and $u_{0}$ decreases, the ring pattern of $v$ is no more persist so that it splits into several pieces of spots and each small spot moves outerward, splitting into two smaller spots, and the resulting $w(t, x)$ exhibits a branched pattern. It is interesting that splitting process of one spot into two in $v$ implies that tip-splitting of branches in $w$. Of course, after large time, as was noted before, all of the spots dissapear but the history of $v$, that is, $w$ clearly records a pattern with branched structure. As $d$ decreases further, keeping $u_{0}$ fixed, splitting of spots in $v$ occurs very irregularly and the resulting branched pattern of $w$ bocomes more complex and exhibits a DLA-like ones(Fig. 4). Finally, when $d$ and $u_{0}$ are small, $v$ does neither grow nor split and fades out and $w$ forms a small patterns with sparse fingers.

The above numerical results indicate that even if $v$ becomes extinction after large time, $w$ given by (2-9) exhibits very complex spatial pattern. In ordert to analyze it, we need the information of the whole time-histroy of $v$. One notices that this is exactly similar to the bacterial colny patterns. Quite recently it has been reported that similar complex patterns to the aboves also appear in combustion under microgravity. Unfortunately, any analytic methods have not been developed to undeerstand these problems. This is a future study for us.

3. Competition-diffusion systems and singular limits. Understanding of spatial and/or temporal behaviors of ecologically interacting species is a central problem in population ecology. As for competitive interaction of ecological species, problems of coexistence and exclusion have been theoretically investigated by using different types of mathematical models. Especially, a variety of RD equations have been proposed to study spatial segregation of competing species. Quite recently, in order to understand the evolutional behavior of spatially segregating regions of competing species, the methods which are called spatial segregation limits have been successfully developed. These enable us to derive evolutional equations describing the boundaries of spatially segregating patterns of competing species. In some situations, the derived equations are described by new types of free boundary problems.

As a well known model in mathematical ecology, we are concerned with a RD system of Gause-Lotka-Volterra type. Let $u_{i}(t, x)$ be the population density of the $i$-th species $U_{i}(i=1,2, \cdots, N)$ at time $t>0$ and the position $x \in \Omega$, where $\Omega$ is a bounded domain in $R^{2}$. The resulting system for $u_{i}(i=1,2, \cdots, N)$ is given by

$$
u_{i t}=d_{i} \Delta u_{i}+f_{i}\left(u_{1}, u_{2}, \cdots, u_{N}\right)(i=1,2, \cdots, N) \quad t>0, x \in \Omega .
$$


Here $d_{i}$ is the diffusion rate of $u_{i}$ and $f_{i}=\left(r_{i}-\sum_{i, j=1}^{N} a_{i j} u_{j}\right) u_{i}$, where $r_{i}$ is the intrinsic growth rate, $a_{i i}$ and $a_{i j}$ are respectively the intraspecific and the interspecfic competition rates $(i, j=1,2, \cdots, N)$. All of the rates are positive constants. We impose the zero-flux boundary conditions to (3-1)

$$
\frac{\partial u_{i}}{\partial \nu}=0(i=1,2, \cdots, n) \quad t>0, x \in \partial \Omega
$$

where $\nu$ is the outerward normal unit vector on $\partial \Omega$. The initial conditions are

$$
u_{i}(0, x)=u_{0 i}(x)(i=1,2, \cdots, N), x \in \Omega \text {. }
$$

The simplest system of (3-1) is the case with $N=2$, that is,

$$
\begin{aligned}
& u_{1 t}=d_{1} \Delta u_{1}+\left(r_{1}-a_{1} u_{1}-b_{1} u_{2}\right) u_{1} \\
& u_{2 t}=d_{2} \Delta u_{2}+\left(r_{2}-b_{2} u_{1}-a_{2} u_{2}\right) u_{2}
\end{aligned} \quad t>0, x \in \Omega
$$

With the same boundary and initial conditions as (3-2), (3-3), qualitative properties of non-negative solutions of (3-4) have been intensively studied by many authors. The first remark is that the stable attractor of (3-4) consists of equilibrium solutions only[2]. By this information, for the study of asymptotic behavior of solutions, we may be concerned with existence and stability of non-negative equilibrium solutions.

In order to study the asymptotic behavior of solutions, we ecologically assume the situation where two species are strongly competing, that is,

$$
\frac{a_{1}}{b_{2}}<\frac{r_{1}}{r_{2}}<\frac{b_{1}}{a_{2}}
$$

which indicates that stable constant equilibrium solutions are $\left(u_{1}, u_{2}\right)=\left(r_{1} / a_{1}, 0\right)$ and $\left(0, r_{2} / a_{2}\right)$ only. These solutions mean that only one of the competing species survives and the other is extinct. When the domain $\Omega$ is convex, any non-constant equilibrium solutions are unstable, even if they exist[13]. In other words, stable equilibria are only $\left(r_{1} / a_{1}, 0\right)$ and $\left(0, r_{2} / a_{2}\right)$, which ecologically indicates that two strongly competing species can never coexist in any convex habitats. This is called Gause's competitive exclusive principle in ecology. On the other hand, if $\Omega$ is not convex, the structure of equilibrium solutions is not so simple but depends on the shape of $\Omega$. If $\Omega$ takes suitable dumb-bell shape, for instance, there exist stable non-constant equilibrium solutions, which exhibit spatial segregation of the two competing species in a sense that one region is nearly occupied by the species $U_{1}$, while the other is by $U_{2}$, that is, two competing species possibly coexist, if the habitat is suitably nonconvex[14]. Integrating the aboves, one notices that there occurs bifurcation phenomenon of equlibrium solutions when the domain-shape changes. We do not touch with this topic here but the reader should refer to [15].

On the other hand, for multi-competing species case, the situation drastically changes. Even if we restrict ourselves to the diffusionless system of (3-1), the solution structures of the resulting ODE system are complicated, where a variety of periodically and aperiodically solutions coexist, in addition to equilibria, depending on parameters $r_{i}$ and $a_{i j}$ ([16] and the references therein). There has been little work for the RD system (3-1) with $N \geq 3$, except for some results[17]. 
3.1. Spatial segregating limits of two competing species model. For (3-4), we already know the asymptotic behavior of solution $\left(u_{1}(t, x), u_{2}(t, x)\right)$, if the domain is convex, that is, the solution tends to either $\left(r_{1} / a_{1}, 0\right)$ and $\left(0, r_{2} / a_{2}\right)$. Even so, from the viewpoint of ecoligical application, it is required to know the transient behavior of solutions. For this purpose, the methods which are called spatial segregation limits have been developed. Let the diffusion rates $d_{1}$ and $d_{2}$ be sufficiently small or all of the other rates $r_{i}, a_{i}$ and $b_{i}(i=1,2)$ be sufficiently large and satisfy (3-5). Then (3-4) is rewritten as

$$
\begin{aligned}
& u_{1 t}=\varepsilon^{2} \Delta u_{1}+\left(r_{1}-a_{1} u_{1}-b_{1} u_{2}\right) u_{1} \\
& u_{2 t}=d \varepsilon^{2} \Delta u_{2}+\left(r_{2}-b_{2} u_{1}-a_{2} u_{2}\right) u_{2}
\end{aligned} \quad t>0, x \in \Omega
$$

in which $\varepsilon$ is a small parameter. It is expected that there occur internal layers which divide the subregions $\Omega_{1}(t)=\left\{x \in \Omega \mid\left(u_{1}(t, x), u_{2}(t, x)\right) \approx\left(r_{1} / a_{1}, 0\right)\right\}$ and $\Omega_{2}(t)=$ $\left\{x \in \Omega \mid\left(u_{1}(t, x), u_{2}(t, x)\right) \approx\left(0, r_{2} / a_{2}\right)\right\}$, which ecologically mean segregating regions of competing species. To study the time evolution of $\Omega_{i}(t)(i=1,2)$, we take the limit $\varepsilon \downarrow 0$ in (3-6) so that the initernal layers become sharp interfaces, say $\Gamma(t)$. Using the singular limit analysis, we obtain the evolution equation to describe the motion of the interface $\Gamma(t)$ can be derived as follows[18]:

$$
V(t)=c-\varepsilon L(d) \kappa
$$

where $V$ is the normal velocity of the interface. $L(d)$ is some positive constant depending on $d$ such that $L(1)=1$ and $c$ is the velocity of the one dimensional travelling front solution $\left(u_{1}, u_{2}\right)(x-c t)$ of

$$
\begin{aligned}
& u_{1 t}=u_{1 x x}+\left(r_{1}-a_{1} u_{1}-b_{1} u_{2}\right) u_{1} \\
& u_{2 t}=d u_{2 x x}+\left(r_{2}-b_{2} u_{1}-a_{2} u_{2}\right) u_{2}
\end{aligned} t>0,-\infty<x<\infty
$$

with the boundary conditions

$$
\left(u_{1}, u_{2}\right)(t,-\infty)=\left(r_{1} / a_{1}, 0\right) \text { and }\left(u_{1}, u_{2}\right)(t, \infty)=\left(0, r_{2} / a_{2}\right)
$$

The velocity $c$ of the travelling front solution of (3-8), (3-9) is unique for fixed values of $r_{i}, a_{i}$ and $b_{i}(i=1,2)[19]$. Quite recently, explicit forms of such travelling front solutions have been found where the dependency of parameters on velocity can be explicitly obtained[20].

Another spatial segregating limit can be discussed where the interspecific competition rates $b_{1}$ and $b_{2}$ are very large[21]. To study this situation, it is convenient to rewrite (3-4) as

$$
\begin{aligned}
& u_{1 t}=d_{1} \Delta u_{1}+r_{1}\left(1-u_{1}\right) u_{1}-b u_{1} u_{2} \\
& u_{2 t}=d_{2} \Delta u_{2}+r_{2}\left(1-u_{2}\right) u_{2}-\alpha b u_{1} u_{2}
\end{aligned} \quad t 0, x \in \Omega
$$

where $b$ and $\alpha$ are positive constants. We assume that $b$ is very large and all other parameters are of order $O(1)$. The coeffcient $\alpha$ is the competition rates between two 
species $u_{1}$ and $u_{2}$. If $\alpha>1$, then $u_{1}$ has competitive advantage over $u_{2}$. while if $\alpha<1$, the situation is reversed. When $b$ is sufficiently large, in other words, the interspecific competiton is very strong, one can expect that $u_{1}$ and $u_{2}$ have disjoint supports (habitats) with only curves which separate the habitats of the competing species. We now derive the time evolution equation of the supports of $u_{1}$ and $u_{2}$, taking the limit $b \uparrow \infty$. The limiting system can be described by a free boundary problem which is a classical two phase Stefan-like problem with reaction terms. Let $\Gamma(t)$ be the interface which separates the two subregions $\Omega_{1}(t)=\left\{x \in \Omega \mid u_{1}(t, x)>0, u_{2}(t, x)=0\right\}$ and $\Omega_{2}(t)=\left\{x \in \Omega \mid u_{1}(t, x)=0, u_{2}(t, x)>0\right\}$ in $\Omega$. Then $u_{1}$ and $u_{2}$ satisfy

$$
\begin{array}{ll}
u_{1 t}=d_{1} \Delta u_{1}+r_{1}\left(1-u_{1}\right) u_{1} & t>0, x \in \Omega_{1}(t) \\
u_{2 t}=d_{2} \Delta u_{2}+r_{2}\left(1-u_{2}\right) u_{2} & t>0, x \in \Omega_{2}(t)
\end{array}
$$

$$
\frac{\partial u_{i}}{\partial \nu}=0(i=1,2) \quad t>0, x \in \partial \Omega
$$

On the interface,

$$
u_{1}(t, x)=0, u_{2}(t, x)=0 \quad t 0, x \in \Gamma(t)
$$

and

$$
0=-\alpha d_{1} \frac{\partial u_{1}}{\partial \zeta}-d_{2} \frac{\partial u_{2}}{\partial \zeta}, \quad t 0, x \in \Gamma(t)
$$

where $\zeta$ is a unit vector normal to $\Gamma(t)$. The initial conditions are given by

$$
u_{i}(0, x)=u_{i 0}(x)(i=1,2) \quad x \in \Omega
$$

and their supports are separated by

$$
\Gamma(0)=\Gamma_{0}
$$

The problem is to find unknown functions $\left(u_{1}(t, x), u_{2}(t, x)\right)$ and $\Gamma(t)$ which satisfy $(3-11) \sim(3-16)$. If this problem can be solved, the interface $\Gamma(t)$ determines the segregating patterns between the two strongly competing species.

3.2. Dynamics of triple junctions arising in the three competing species model[22]. We consider (3-1) with $N=3$ in two dimensions, that is,

$$
u_{i t}=d_{i} \Delta u_{i}+f_{i}\left(u_{1}, u_{2}, u_{3}\right) \quad t>0, x \in \Omega \text { in } R^{2}
$$

where $f_{i}\left(u_{1}, u_{2}, u_{3}\right)=\left(r_{i}-a_{i 1} u_{1}-a_{i 2} u_{2}-a_{i 3} u_{3}\right) u_{i}(i=1,2,3)$. We simply write it as

$$
U_{t}=D \Delta U+F(U) \quad t>0, x \in \Omega
$$


where $U=\left(u_{1}, u_{2}, u_{3}\right), D$ is the $3 \times 3$ diagonal matirx with elements $\left\{d_{i}\right\}(i=1,2,3)$ and $F=\left(f_{1}, f_{2}, f_{3}\right)$. We first consider the diffusionless system of $(3-17)$

$$
U_{t}=F(U) \quad t>0,
$$

Assume that $(i \neq j)$ are large comparing with others $a_{i i}$ to require that $P_{1}=$ $\left(r_{1} / a_{11}, 0,0\right), P_{2}=\left(0, r_{2} / a_{22}, 0\right)$ and $P_{3}=\left(0,0, r_{3} / a_{33}\right)$ are stable and other critical points are all unstable, that is, three species are in strong competition. It is thus shown that almost all non-negative solutions $U(t)$ of $(3-20)$ tend to one of $P_{i}(i=1,2,3)[23]$. This ecologically implies that one of the competing species can survive and the other two are extinct. We remark that the dynamics of $U(t)$ is extremely simple. Under the non-coexistence situation stated above, we consider what kind of patterns RD system (3-17) generates by the interplay of diffusion and reaction. In order to study this problem, we assume that the diffusion rates $D$ is so sufficiently small, that is, $D=\varepsilon \bar{D}$ with a sufficiently small parameter $\varepsilon$, where $\bar{D}$ is a diagonal matrix with elements $\left\{\overline{d_{i}}\right\}(i=1,2,3)$. The resulting system from $(3-18)$ is written as

$$
U_{t}=\varepsilon^{2} D \Delta U+F(U) \quad t>0, x \in \Omega,
$$

where we wrote $\bar{D}$ as the original $D$. The initial and boundary conditions are

$$
U(0, x)=U_{0}(x) \quad x \in \Omega,
$$

and

$$
\frac{\partial U}{\partial \nu}=0 \quad t>0, x \in \partial \Omega
$$

where $\nu$ is the outward normal unit vector on $\partial \Omega$. Since $\varepsilon$ is sufficiently small, one can expect that the behavior of solutions of (3-4) (3-6) essentially consists of two stages; The first stage is the occurrence of internal layer regions (when $\varepsilon$ tends to zero, they become interfacial curves), which generally divide the domain $\Omega$ into three subdomains $\Omega_{1}, \Omega_{2}$ and $\Omega_{3}$ where the solution $U(t, x)$ is close to one of $P_{i}(i=1,2,3)$. This indicates the appearance of spatial segregation of three competing species with triple junctions where three interfacial curves meet. The second stage is the motion of interfacial curves with triple junctions, that is, the dynamics of segregating patterns.

We numerically consider (3-20) (3-22) in a rectangular domain $\Omega$. The first stage is the occurrence of segregating patterns of $\left(u_{1}, u_{2}, u_{3}\right)$ so that the domain $\Omega$ is clearly divided into three subdomains $\Omega_{1}, \Omega_{2}$ and $\Omega_{3}$, which are separated by interfacial curves with triple junctions. Consider the second stage. The first case is the completely symmetric case where $d_{i}=d, a_{i i}=a, a_{i j}=b(i=1,2,3)$. The dynamics of segregating pattern changes slowly. We note the following two points:

(i) if interfacial curves are almost straight, they move very slowly;

(ii) angles between any two neighboring interfacial curves are equal.

This angle condition of interfaces can be intuitively understood by the fact that three competing species possess completely symmetric property.

The next cases are non-symmetric. The first example is a semi-symmetric case where $d_{i}=d(i=1,2,3), a_{12}=a_{21}=b_{1}, a_{23}=a_{32}=b_{2}, a_{31}=a_{13}=b_{3}$, but $b_{1}, b_{2}$ 
and $b_{3}$ are not necessarily equal. The dynamics of segregating pattern is qualitatively similar to the above symmetric case except for the angles of interfaces at triple junction areas, which seem to be not necessarily equal.

The second is the case with ordering property where $a_{12}<a_{21}, a_{13}<a_{31}$ and $a_{23}<a_{32}$, that is, the species $U_{1}$ is the strongest of the three. The resulting dynamics of pattern is much faster than the previous two cases and, as is easily expected, the domain is gradually occupied by the strongest species $U_{1}$.

The third is a symmetrically cyclic case where $a_{12}=a_{23}=a_{31}=b$ and $a_{21}=$ $a_{32}=a_{13}=b^{\prime}$ with $b \neq b^{\prime}$. As the cyclic property suggests, one can expect that there appear stationary rotating spiral patterns with three arms(Fig. 5).

Finally, the fourth is a general case with cyclic property where $a_{12}<a_{21}, a_{23}<$ $a_{32}$ and $a_{31}<a_{13}$. There is no longer any stationary rotating spirals but complex spatio-temporal patterns with several clustering spirals, where each spiral seems to be steadily rotating in a vicinity of triple junction areas(Fig. 6).

We have observed that segregating patterns of three competing species are drastically different from the ones of two competing species, that is, even if $\Omega$ is convex domain in $R^{2}$, it is possible for the three species to coexist but the resulting pattern is not stationary but very dynamic.

4. Cocluding remarks. We have specified two types of systems among many $\mathrm{RD}$ ones. One is resource-consumer systems without feeding process where the reactants becomes spatially homogenoeus for large time, and the other is a competetiondiffusion system where the two competing species model possess no stable spatially non-constant equilibrium solutions For these reasons, these two systems had been left from pattern formation viewpoints. However, we emphasize in this lecture that for the former system, transient behaviors of solutions is very important and its record exhibits compelx spatial patterns, and for the latter, the three species model exhibits spatio-temporal pattern with triple junction dynamics. Unfortunately, the analysis of these systems have not been developed and it is a future study to us.

Acknowledgements: The author would like to thank N. Dancer, D. Hilhorst, S.-I. Ei, R. Ikota, M. Matsushita, L. A. Peletier, H. Sakaguchi, D. Ueyama for jointly working with him for those topics in this lecture.

\section{REFERENCES}

[1] Conway, E., Hoff, D. AND Smoller, J., Large time behavior of solutions of systems of nonlinear reaction-diffusion equations, SIAM J. Appl. Math., 35 (1978), pp. 1-16.

[2] HiRsch, M. W., Differential equations and convergence almost everywhere of strongly monotone semiflows, PAM Technical Report, Univ. California, Berkley (1982).

[3] Casten, R. G. AND Holland, C., J., Instability results for reaction-diffusion equations with Neumann boundary conditions, J. Differential Equations, 27 (1978), pp. 266-273.

[4] Matano, H., Asymptotic behavior and stability of solutions of seminlinear diffusion equations, Pub. R.I.M.S., Kyoto Univ., 15 (1979), pp. 401-454.

[5] Turing, A., The chemical basis of morphogenesis, Phil. Trans. Roy. Soc. Lond., B237 (1952), pp. 23-72.

[6] Hodgkin, A. L. And Huxley, A. F., A qualitative description of membrane current and its application to conduction and excitation in nerve, J. Physiol., 117 (1952), pp. 500-544.

[7] FitzHugh, R., Impulses and physiological states in models of nerve membrane, Biophys. J., 1 (1961), pp. 445-466.

[8] Nagumo, J., Arimoto, S. And Yoshizawa, S., An active pulse transmission line simulating nerve axon., Proc. IRE, 50 (1962), pp. 2061-2070.

[9] Matsushita, M. ET AL., Interfaces growth and pattern formation in bacterial colonies, Physica A, 249 (1998), pp. 517-524. 
[10] Mimura, M., Sakaguchi, H. AND Matsushita, M., Reaction-diffusion modelling of bacterial colonies, Physica A, 282 (2000), pp. 283-303.

[11] Gray, P. AND ScotT, S. K., Autocatalytic reactions in the isothermal, continuous stirred tank reactor, Chem. Eng. Sci., 39 (1984), pp. 1087-1097.

[12] Hoshino, H., On the convergence properties of global solutions for some reaction-diffusion systems under neumann boundary conditions, Diff. Integral Eqs., 9 (1996), pp. 761-778.

[13] Kishimoto, K. AND WeIBERGER, H., The spatial homogeneity of stable equlibria of some reaction-diffusion system on convex domains, J. Differential Equations, 58 (1985), pp. $15-21$.

[14] Matano, H., ANd Mimura, M., Pattern formation in competition-diffusion systems in nonconvex domains, Pub. R.I.M.S., Kyoto Univ., 19 (1983), pp. 1049-1079.

[15] Mimura, M., EI, S.-I. AND FANG, Q., Effect of domain-shape on coexistence problems in a compeititon-diffusion system, J. Math. Biol., 29 (1991), pp. 219-237.

[16] Mimura, M. AND KAN-ON, Y., Predator-mediated coexistence and segregation structures, Studies in Math. Its Appl., 18 (1986), pp. 129-155.

[17] Mimura, M. AND Fife, P., A 3-component system of competition and diffusion, Hiroshima Math. J., 16 (1986), pp. 189-207.

[18] EI, S.-I. AND YANAGIDA, E., Dynamics of interfaces in competition-diffusion systems, SIAM Appl. Math., 54 (1994), pp. 1355-1373.

[19] KAN-ON, Y., Existence of travelling wave solutions for competition-diffusion equations, SIAM J. Math. Anal., 26 (1995), pp. 340-363.

[20] Rodrigo, M. AND Mimura, M., Exact solutions of competititon-diffusion systems, Hiroshima Math. J., 30 (2000), pp. 257-270.

[21] Dancer, E. N., Hilhorst, D., Mimura, M. And Peletier, L. A., A spatial segregation limit of a competition-diffusion systems, Euro. J. Appl. Math., 10 (1999), pp. 97-115.

[22] Ei. S.-I., IкотA, R. AND MimuRA, M., Segreagating partition problem in competition-diffusion systems, Interface and Free Boundaries, 1 (1999), pp. 57-80.

[23] VAN DEN Driessche, P. AND ZeEman, Three-dimensional competitive Lotka-Volterra systems with no periodic orbits, M. L.: SIAM J. Appl. Math., 58 (1998), pp. 227-234.

\section{Figure caption}

Fig. 1 Phase diagram of colonies of Bacillus sbtilis

Fig. 2 DLA-like pattern

Fig. 3 Numerical DLA-like pattern

Fig. 4 Dynamics of numerical solutions of (2-8) with $m=2$ and $n=1$

Fig. 5 Stationary rotating spirals with three arms

Fig. 6 Spatio-temporal patterns with clustering spirals 


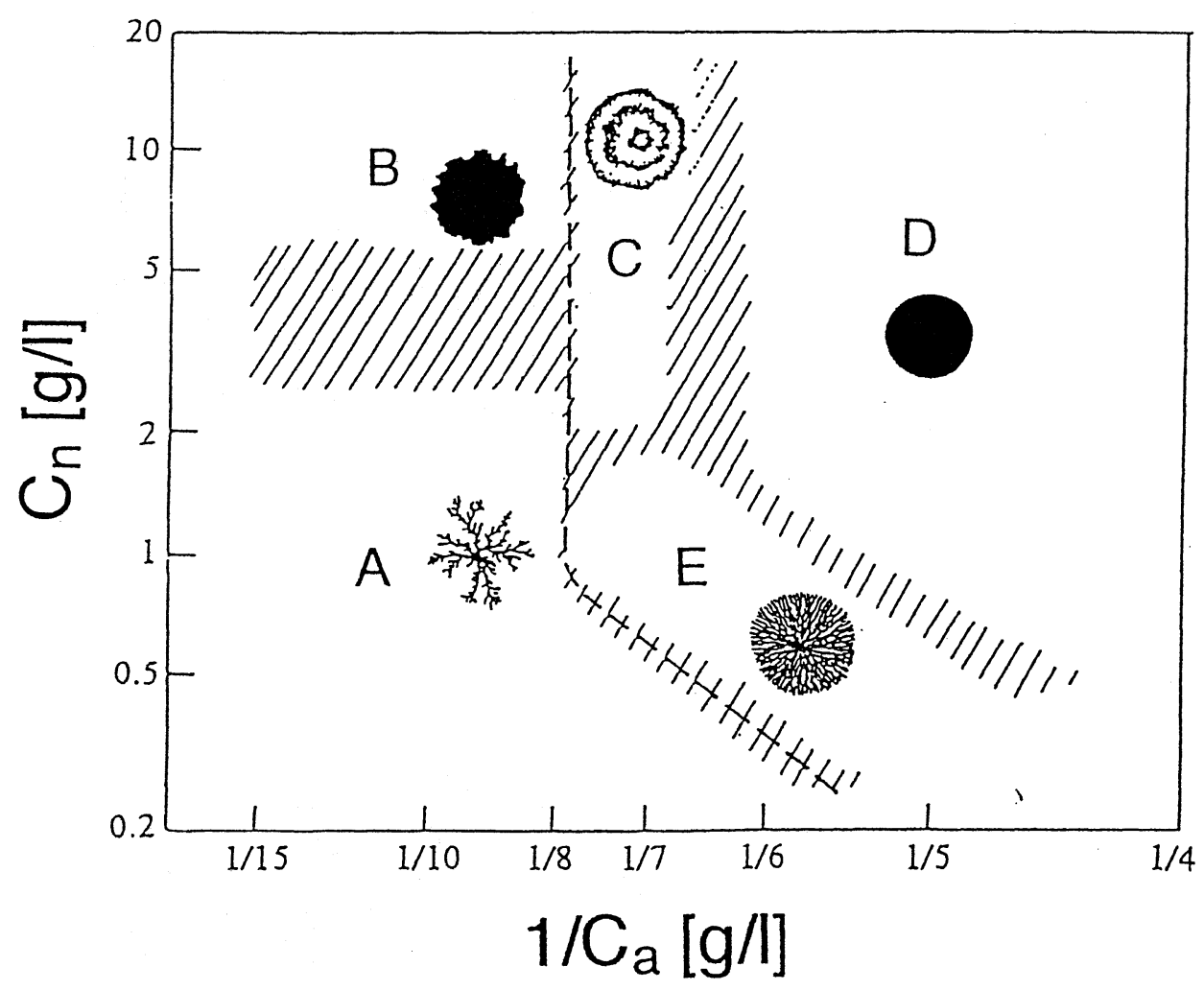

Fig. 1 


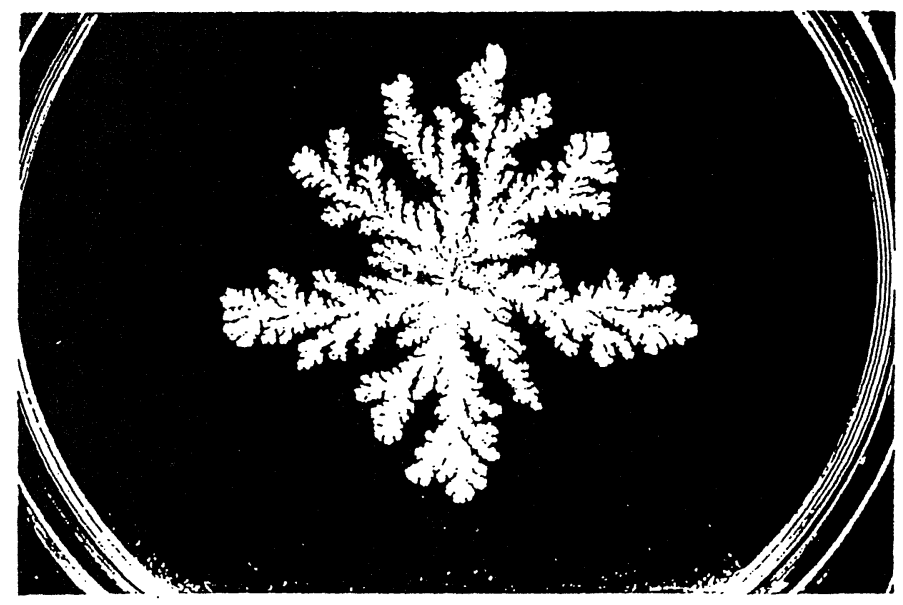

Fig. 2

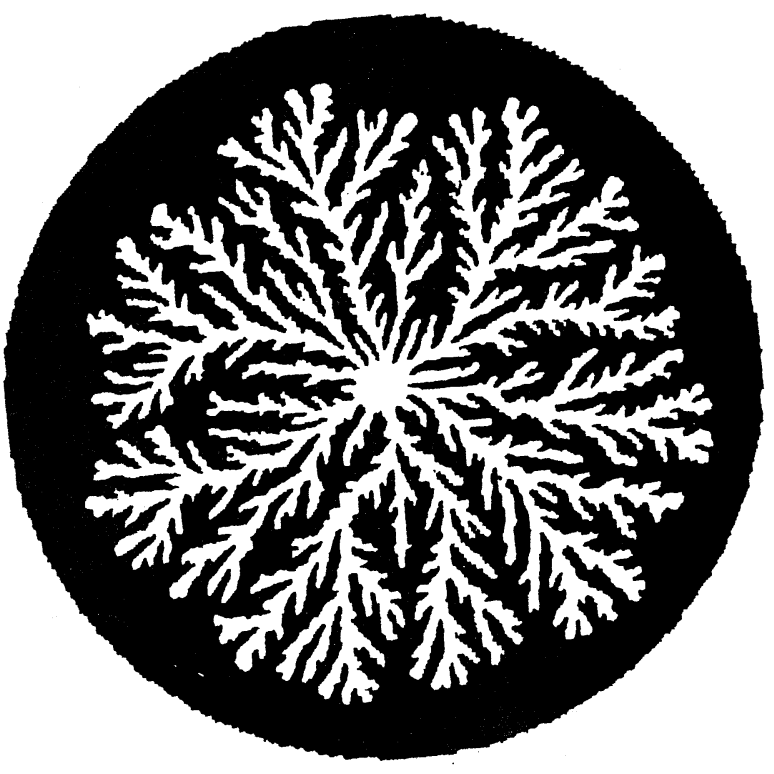

Fig. 3 
$\Omega=(2000 \times 2000), D=10, \beta=0.025, U_{0}=1$
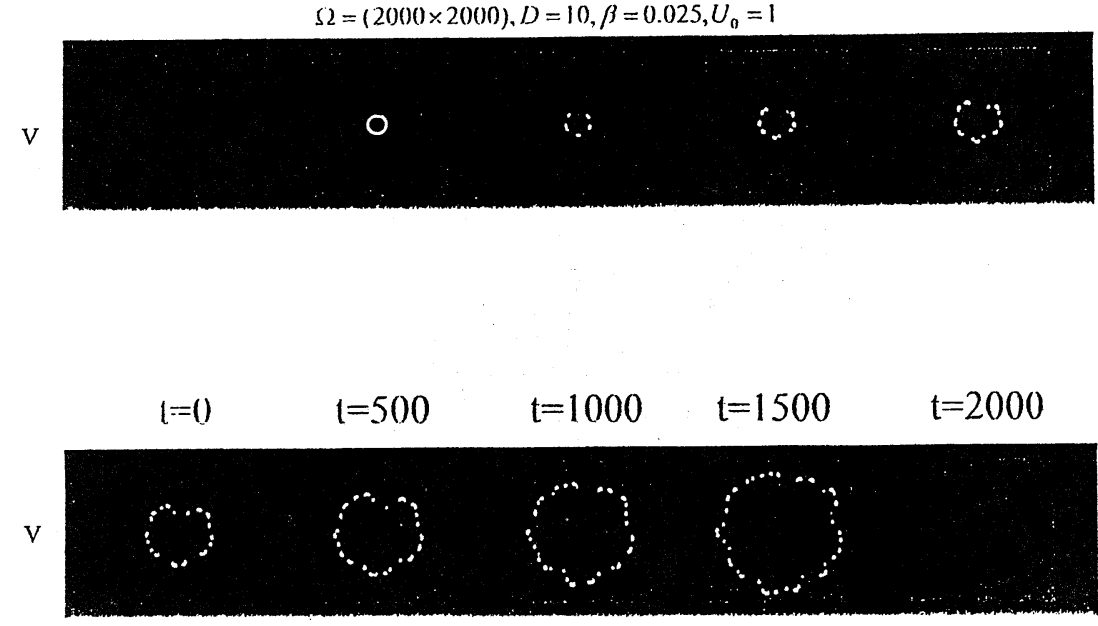

$t=3000 \quad t=4000 \quad t=5000 \quad t=6000 \quad t=12500$
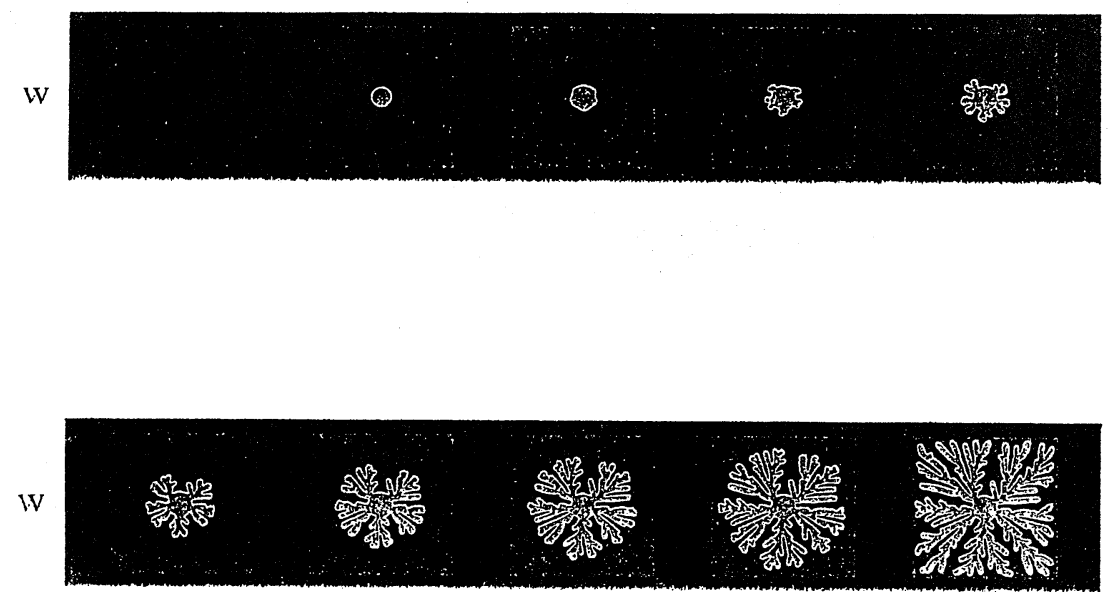

Fig. 4 


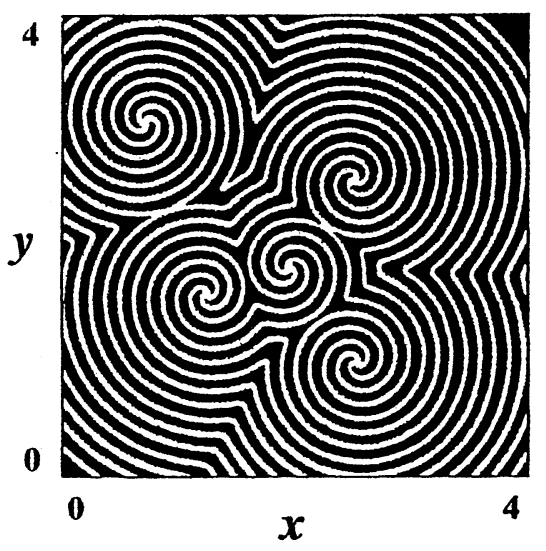

Fig. 5

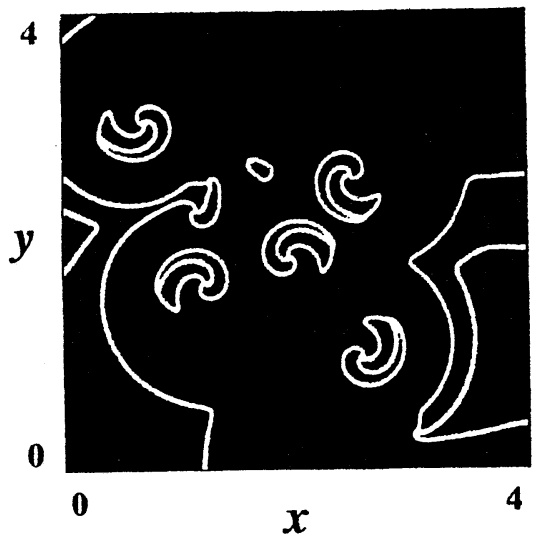

Fig. 6 